\title{
The Portuguese Manufacturing Sector during 2013-2016 after the Troika Austerity Measures
}

\author{
Kelly P. Murillo ${ }^{a}$ \\ EugÉnIO M. ROCHA ${ }^{\mathrm{b}}$ \\ Received: 14.03.2018; Revised: 01.04.2018; Accepted: 03.05.2018
}

\begin{abstract}
This work studies the effects of the Troika austerity measures on the Portuguese manufacturing firms in terms of efficiency scores. We adopted a non-parametric approach, which combines multidirectional efficiency analysis with other techniques, to examine two empirical hypotheses after the financial crisis and corresponding intervention of the Troika measures: (a) the performance of firms in the manufacturing sector has improved; (b) the manufacturing sector significantly acquired long-term debt but use it in an efficient way. Our results show that validation of the first hypothesis heavily depends on the firm size, and the second hypothesis is correct only with respect to long-term debt acquiring. In fact, some sectors have managed to maintain an acceptable level of efficiency, according to the circumstances, however, most of them have showed some inefficiency in the management of resources and less than $10 \%$ have been able to overcome the difficulties emerged after the intervention of the Troika. A common tool to overcome a crisis is the acquisition of long-term debts, which was done by $77 \%$ of firms; but with a lower gain, since it was the most efficient input resourced used. On the contrary, our results show that the number of employees and total assets are better leverage to maintain efficiency.
\end{abstract}

JEL codes: C14, C38, D22, L60

Keywords: Multidirectional efficiency analysis, Clustering analysis, Manufacturing sectors, World financial crisis, Austerity measures

\section{Introduction}

Due to the financial crisis started on 2008, many countries suffered strong economic deceleration and rising unemployment. There were strong consequences on industrial relations in all the $27 \mathrm{EU}$ Members. In fact, the industrial relations had a decrease in the overall volume of bargaining, an increase in the number of agreements not being renewed, and a trend towards shorter agreements. There is also much evidence of pay pauses, pay freezes, pay cuts and working time reductions (Broughton \& Welz, 2013).

Some countries have received financial support to overcome financial difficulties. In fact, Greece, Ireland and Portugal are the three European countries that have requested financial

${ }^{a}$ Corresponding author. Center for Research and Development in Mathematics and Applications (CIDMA) and Department of Mathematics, University of Aveiro, Aveiro, Portugal. e-mail: kellymurillo@ua.pt

b Center for Research and Development in Mathematics and Applications (CIDMA) and Department of Mathematics, University of Aveiro, Aveiro, Portugal. e-mail: eugenio@ua.pt 
rescue to the team composed of the International Monetary Fund (IMF), the European Central Bank (ECB) and the European Commission (EC) (the so-called Troika). The Troika in Portugal was implemented between May 2011 and May 2014, by including several austerity policies such as tax hikes and salary cuts. The IMF measures imposed on the Portuguese Government, after the request for financial redemption to the EC on 6 April 2011, aimed at reducing the deficit to $5.9 \%$ in $2011,4.5 \% 2012$ and $3 \%$ by 2013 .

The European Union and the IMF's financial assistance program for Portugal was around EUR 78 billion and has been implemented for three years, including measures in taxes, salaries, public function, unemployment subsidy and companies. In the last years, we find some papers in the literature analyse the role of Portugal in the crisis and subsequent reaction to the intervention of the Troika, e.g. the work of Eichenbaum et al. (2016), which evaluates the IMF's role in the 2011 program for Portugal and its surveillance of the Portuguese economy in the preceding decade. Blattner et al. (2016) and Abreu et al. (2013) are the other examples.

The main aim of this paper is to study two possible effects of the Troika austerity measures on the manufacturing sector at three distinct stages: efficiency levels; efficiency patterns; and efficiency determinants. We use a dataset of Portuguese manufacturing firms survived between 2014 and 2016 .

There is a general opinion that Portugal has been profoundly affected by the global financial crisis, the impact of the recession and subsequent austerity on different economic sectors. A real study of the economic situation in Portugal must involve before, during and after the crisis. In previous works, the authors of this article studied the efficiency of the Portuguese firms on three time periods: pre-crisis (2006-2008), pre-Troika (2009-2011) and Troika (2012-2013). This study is focused on the post-Troika (2014-2016) period. The data is divided into seven sub-sectors (described in detail in the Section 3.1), since the effects over the efficiency of the manufacturing industry may not have been manifested equally for all sectors.

The most common technique for measuring the efficiency of Decision Making Units (DMUs) is the Data Envelopment Analysis (DEA) proposed by Banker et al. (1984). The DEA approach has been widely investigated and applied to many fields and industries. However, DEA presents a range of issues that needed to be taken care of (e.g. non-homogeneous units, economies of scale, percentages and/or normalised data) that significantly limit its practice, see Dyson et al. (2001). Moreover, DEA cannot be used to assess changes in inefficiency patterns, since it is based on radial contractions on all inputs and undesirable outputs and radial expansions on all desirable outputs. In order to counteract all the difficulties mentioned above (see Section 3.3 for details), in this work we use a more general nonparametric deterministic method for measuring efficiency, namely a model based in the Multidirectional Efficiency Analysis (MEA), proposed by Bogetoft and Hougaard (1999). The advantages of the use of MEA, among the others, are it allows to investigate changes in efficiency patterns; the input reduction and output expansion reference points are selected proportionally to the possible improvements in efficiency identified when considering the improvement potential separately in each input variable and output variable. Many relevant papers in different fields use MEA instead of the traditional DEA. In Wang et al. (2014), MEA is used to evaluate the environmental efficiency of industrial sectors of Chinese major cities. In Bi et al. (2014), a nonradial DEA model with MEA involving undesirable outputs for the measurement of regional energy and environmental efficiency of China's transporta- 
tion sector during the period 2006-2010. The work in Mohd Suhaimi et al. (2017) uses MEA to measure the technical inefficiency of dairy farms and subsequently investigate the factors affecting technical inefficiency in the Malaysian dairy industry. Others examples of applications of the MEA model to examine efficiency patterns can be found in Wang et al. (2013); Asmild et al. (2009); Baležentis and De Witte (2015); Asmild et al. (2016), and Kapelko and Lansink (2017).

For our analysis, firms are divided into groups according to their size/scale by using a sample partition between micro, small, and medium plus large firms, since smaller firms tend to act differently than larger firms and are affected differently in numerous aspects of their economic behaviour. In fact, small firms differ from large firms in taxability, ownership, flexibility, industry, economies of scale, financial market access, and level of information asymmetry, see Scherr and Hulburt (2001) and Vermoesen et al. (2013).

We choose the most meaningful variables to perform a coherent analysis of firm's efficiency, using Principal Component Analysis (PCA) proposed in Pearson (1901), together with a dimensionality test described in Robert and Escoufier (1976), in order to avoid loss of information or the introduction of random noise.

For processing the data and obtain the results presented, we develop a general sDL package based on the R language that is available online at http://sdl-vm2 .mathdir .org/.

The remainder of the paper is laid out as follows. In the next section, a brief overview on Efficiency behaviour of the Portuguese manufacturing firms during the financial crisis and the hypotheses of this study are formulated. Section 3 discusses the MEA approach used in this study, Section 4 presents the main results, which allow to analyse the two hypotheses. In Section 5, some concluding remarks are formulated. At the end, we provide the appendix with the description of the MEA model used.

\section{Review of literature and research hypothesis}

Manufacturing industry in Portugal has been studied from different perspectives. The work of Matias et al. (2015) studies the determinants of capital structure of 1,488 small and medium size Portuguese companies belonging to the manufacturing sector. The analysis of a 2004-2011 panel data suggests that trade-off and pecking order theories are not mutually exclusive in explaining capital structure decisions. Pechankova (2016) studied that in which way the Portuguese manufacturing industry, and its respective sectors, has a direct and indirect impact on the creation of value added and employment and how this impact can be measured. The work of Cabral (2007) offers an in-depth analysis of Portuguese firms comprising entry and exit decisions, firm size, productivity and distortions to economic activity. In Amador (2011) a non-parametric approach is adopted to explore the relation between size, capital intensity and productivity in a set of four Portuguese manufacturing sectors: "food and beverages, clothing", "manufactured non-metallic mineral products" and "metallic products, except machinery and equipment". Amador and Opromolla (2009) states that the structure of the Portuguese textile and clothing sectors is based on small medium firms and the analysis of firm-level data reveals some reduction in their average size from 1996 to 2005. In Nogueira et al. (2017), the Portuguese manufacturing industries are divided into high-tech and low-tech and study the approach that best justifies the wage gap, for the period between 2007 and 2014. 
Specifically on the impact of the crisis, the majority of the works in the literature analyse the situation of the Portuguese economy in the general context without taken great focus in the manufacturing industry. Pedroso (2014) assessed how Portugal's strategy to deal with the international economic crisis has affected the country's economy and social situation. In Eichenbaum et al. (2016), the IMF's role is evaluated in the 2011 program and its surveillance of the Portuguese economy in the preceding decade. In Gurnani (2016), the origins of the financial crisis in Portugal are reviewed. It provides an overview of the program and its short-term impacts. The work of González (2014) outlines the main changes that the Portuguese labour market has suffered from following the deterioration of financial situation and particular attention is paid to the gender (and biases) of the crisis. Petmesidou and Guillén (2015) reviewed reform trends prior to and during the crisis in order to highlight convergent and divergent paths among Southern European countries, which hit the hardest and the longest by the post-2008 economic crisis. For other interesting works see Costa (2012); Heyes and Hastings (2016); Matos et al. (2015) and Blattner et al. (2016).

\subsection{Efficiency behaviour of the Portuguese manufacturing firms during the fi- nancial crisis}

During the international economic crisis, the Portuguese economy, in general, had undergone some major changes, verifying an increase in closures of firms and an escalation in the number of insolvencies, which reached the highest value since 2007. According to the Portuguese National Statistics Institute, it has been reported that industrial production in Portugal had an average decreasing of $-0.63 \%$, from 2005 to 2015 . However, some manufacturing firms show that the degree of reaction to the crisis is not necessarily negatively related to productivity. These firms show greater capability to adapt to changes either within their organization and/or in its environment.

In previous works, the authors of this article studied the efficiency of the Portuguese manufacturing firms on three time periods: pre-crisis (2006-2008), pre-Troika (2009-2011) and Troika (2012-2013). The results show that the efficiency of firms decreased after the crisis for most of the subsectors except for manufacture of textiles and transport equipment. These subsectors showed the capacity to adapt to various changes brought by the crisis. The results also show that due to the financial crisis, the manufacturing sector acquired longterm debt deliberately. Moreover, even with long-term debts acquired, many firms failed in improving their productivity, indicating that the resource was used to support unfavourable situations and not to improve their efficiency globally. The food subsector was one of the few sectors that managed to maintain a stable performance during and after the crisis. Paradoxically, although it was not pointed out, among the subsectors that improved their efficiency after the crisis, the food subsector did not suffer major decay in their economicallyfinancial management. Furthermore, in this subsector there was an increase in the number of employees in the Troika period and, some firms improved the solvency without resorting to huge debts.

\subsection{Research hypothesis}

The weak financial capacity, poor preparation of human resources and the high dependence on external raw materials are some factors that usually negatively influence the strategic performance of the Portuguese manufacturing firms. Over time, it is expected that the negative effects of the European crisis would decrease and the market attains some 
stability, in line with efforts to reduce the losses in all the manufacturing sector.

The purpose of this study is to examine the effects of the financial crisis on efficiency of the manufacturing sector of Portuguese industry in three distinct phases: the levels of efficiency; efficiency standards; and determinants of efficiency. To this end, our study focuses on studying two main hypotheses.

(H1) With the intervention of the Troika, the performance of firms in the manufacturing sector has improved.

In the manufacturing sector, more than in any other sector in Portugal, the changes after the crisis were very noticeable. We intend to understand to what extent the intervention of the Troika has affected the performance of the firms.

(H2) After the financial crisis and corresponding intervention of the Troika, the manufacturing sector significantly acquired long-term debt and used it in an efficient way.

The intervention of the Troika led to tightening measures, in order to avoid as quickly as possible, the impairment in economical-financial management of the Portuguese firms. The acquisition of debt was one of the most common solutions assumed by the manufacturing firms to overcome the difficulties caused during the financial crisis. We intend to understand how the attitude of the manufacturing industry has changed due to the acquisition of the long term debt, after the intervention of the Troika, and how it has affected its efficiency in each of the subsectors.

\section{Data and Methodology}

\subsection{Characterization of the data}

The data set of study is collected from the Amadeus (Bureau van Dijk) database, which comprises financial information and other characteristics of the Portuguese firms operating in the manufacturing sector (NACE Rev.2-Statistical classification of economic activities in the European Community, section C, codes between 1000 and 3399). We considered only the firms with data available for all years in the period 2014-2016 and partitioned the manufacturing sector in seven subgroups as follows (NACE codes are in parentheses):

1) FOOD (1000-1299): Manufacture of food products, beverages and tobacco products - 3,653 firms;

2) TEXT (1300-1599): Manufacture of textiles, apparel, leather and related products - 5,167 firms;

3) MATER (1600-1899, 2200-2599): Manufacture of wood and paper products, and printing; Manufacture of rubber and plastics products, and other non-metallic mineral products; Manufacture of basic metals and fabricated metal products, except machinery and equipment - 2,586 firms;

4) CHEM (1900-2099): Manufacture of coke, and refined petroleum products; Manufacture of chemicals and chemical product - 403 firms;

5) $M E D$ (2100-2199): Manufacture of pharmaceuticals, medicinal chemical and botanical products - 68 firms; 
6) EQUIP (2600-2899, 3100-3399): Manufacture of computer, electronic and optical products; Manufacture of electrical equipment; Manufacture of machinery and equipment n.e.c; Other manufacturing, and repair and installation of machinery and equipment - 3,264 firms;

7) TRANSP (2900-3099): Manufacture of transport equipment - 358 firms.

For this study, we analysed the (technical) efficiency of 46,497 units (i.e. 15,499 firms in three years). For each year and firm, we extracted from the Amadeus database the following 13 variables: (1) number of employees (NE); (2) cash and cash equivalent (CASH); (3) issued share capital (CAPITAL); (4) total assets (TASSETS); (5) long term debt (LTDEBT), defined as the company's total debt due for repayment beyond one year; (6) profit margin (PROFITM), found by multiplying by 100 the result of dividing profit before tax by operating revenue; (7) current liabilities (CLIAB); (8) liquidity ratio (LIQR), defined by the difference between current assets and stocks, divided by current liabilities; (9) solvency ratio (SOLVR), calculated by multiplying by 100 the result of dividing the shareholders' funds and total assets; (10) sales (SALES); (11) EBIT margin (EBITM), calculated by multiplying by 100 the result of dividing the difference between all operating revenues and all operating expenses by operating revenue; (12) EBITDA margin (EBITDAM), found by multiplying by 100 the result of dividing the sum of operating profit and depreciation by operating revenue; and (13) cash flow (CASHFLOW).

\subsection{Multidirectional efficiency analysis}

This work uses MEA to study the effects of the Troika measures on the efficiency of the Portuguese manufacturing sector. The MEA efficiency model was proposed by Bogetoft and Hougaard (1999) and further developed in Bogetoft and Hougaard (2004) and Asmild and Pastor (2010). In what follows, we give a general description of the model and fix notation. Further details are presented in the Appendix A.

Let $n=(c, s, t) \in N$ be a tuple identifying the firm $c \in C$, the sector $s \in S$ and year $t \in T$, which we call a firm/sector/year tuple, and $[m]$ denotes the set $1, \ldots, m$, for some $m \in N$.

We consider that any given tuple $n \in N$ produces $j \in N$ outputs $y_{j}(n), j \in[J]$, using $I \in N$ inputs $x_{i}(n), i \in[I]$, where the first $1<D \leq I$ inputs are the so-called discretionary inputs, i.e. variables that enter into the optimization process, because the non-discretionary inputs are variables that cannot be changed. Hence, $x(n) \in R^{I}$ is the vector of all the inputs and $y(n) \in R^{J}$ is the vector of all the outputs, for a given sector/year tuple $n \in N$. Our dataset $Z=\{z(n)\}_{n \in N}$ is the set of values $z(n)=(x(n), y(n))$ for all $n \in N$.

For a given dataset $\mathrm{Z}$, the MEA score of each $n \in N$ is then defined as

$$
M E A_{z}(n)=\frac{\frac{1}{\gamma^{*}(\bar{n})}-\frac{1}{D} \sum_{i=1}^{D} \frac{x_{i}(\bar{n})-\alpha_{i}^{*}(\bar{n})}{x_{i}(\bar{n})}}{\frac{1}{\gamma^{*}(\bar{n})}+\frac{1}{J} \sum_{j=1}^{J} \frac{\beta_{j}^{*}(\bar{n})-y_{j}(\bar{n})}{y_{j}(\bar{n})}}
$$

where $\alpha_{i}^{*}(\bar{n}), \beta_{j}^{*}(\bar{n})$ and $\gamma^{*}(\bar{n})$ represent the corresponding optimal solutions to the linear optimization problems $P_{i}^{\alpha}(z, n), P_{j}^{\beta}(z, n)$ and $P^{\gamma}\left(z, n, \alpha^{*}, \beta^{*}\right)$, defined in the Appendix A.

The MEA score is obtained by the directional contribution of each input and each output variable. In fact, for the input $i \in[I]$ the contribution in the unit $z(\bar{n})$ is given by 


$$
\operatorname{meff}_{i}(\bar{n})=\frac{x_{i}(\bar{n})-\gamma^{*}(\bar{n})\left(x_{i}(\bar{n})-\alpha_{i}^{*}(\bar{n})\right)}{x_{i}(\bar{n})} \chi_{[D]}(i)
$$

for $i \in[I]$, where $\chi_{[D]}(i)$ is the characteristics function of the set $[\mathrm{D}]$. That means

$$
\chi_{[D]}(i)= \begin{cases}1, & i \in[D] \\ 0, & i \notin[D]\end{cases}
$$

For the outputs $j \in[J]$ the contribution is given by

$$
\operatorname{meff}_{i}(\bar{n})=\frac{y_{j}(\bar{n})}{y_{j}(\bar{n})+\gamma^{*}(\bar{n})\left(\beta_{j}^{*}(\bar{n})-y_{j}(\bar{n})\right)}
$$

for $j \in[J]$. The term $\gamma^{*}(\bar{n})\left(x_{i}(\bar{n})-\alpha_{i}^{*}(\bar{n})\right)$ in Equation (2) represents the input excess for each $i \in[D]$ and the term $\gamma^{*}(\bar{n})\left(\beta_{j}^{*}(\bar{n})-y_{j}(\bar{n})\right)$ in Equation (3) represents the output shortfall for each $j \in[J]$.

One interesting feature of MEA is that the inefficiency of each input can be analysed individually. In fact, based on the input excess (see Appendix A) and using the ideas in Bogetoft and Otto (2011), we calculate the following Inefficiency Index

$$
R_{i}(n)=\frac{\sum_{n=1}^{N} \gamma(n)\left(x_{i}(n)-a_{i}^{*}(n)\right.}{\sum_{n=1}^{N} x_{i}(n)}
$$

for each given input index $i \in[I]$ and tuple $n \in N$. We refer to the inefficiency index to determine the number of times each input is used inefficiently, since our particular interest is to assess if global efficiency can be improved with less inputs.

\subsection{Specific Characteristics of our Model}

Dyson et al. (2001) reported some pitfalls and issues in the implementation of DEA/MEA that need to be avoided, namely, the following aspects are relevant to be mentioned in this work:

- Economies of Scale: A unity may be too small to operate with optimal efficiency or so large that it becomes difficult to manage;

- Percentages and other normalized data: The pitfall occurs when one tries to mix together indices, ratios and percentages, with volumes measures into variable selections.

Using the results in Hollingsworth and Smith (2003), the two difficulties mentioned before are overcome simultaneity in this work, since, we consider the variable returns to scale VRS (see Banker et al. (1984)). In fact, Hollingsworth and Smith (2003) explains why the Banker et al. (1984) formulation of DEA must be used when a ratio model is deployed. They prove that when DEA data are used in the form of ratios, use of the standard CRS (Charnes et al., 1978) model is technically incorrect, and should be rejected in favour of the VRS model.

One of the dilemmas when dealing with relative rankings, it is how to compare the units over time (i.e. different rankings). To clarify this point, we show the following toy example. In Table 1, the efficiencies of three units (A, B and C) are calculated between two periods 
(T1 and T2). The dataset consists of two inputs (X1 and X2) and one output (Y).

Table 1: Efficiency in the two periods

\begin{tabular}{lcccc|ccccc}
\hline Units & X1 & X2 & Y & Eff T1 & Units & X1 & X2 & Y & Eff T2 \\
\hline $\mathrm{A}(\mathrm{T} 1)$ & 1 & 2 & 1 & 1 & $\mathrm{~A}(\mathrm{~T} 2)$ & 3 & 4 & 1 & 1 \\
$\mathrm{~B}(\mathrm{~T} 1)$ & 2 & 1 & 1 & 1 & $\mathrm{~B}(\mathrm{~T} 2)$ & 4 & 3 & 1 & 1 \\
$\mathrm{C}(\mathrm{T} 1)$ & 3 & 3 & 1 & 0.5 & $\mathrm{C}(\mathrm{T} 2)$ & 4 & 4 & 1 & 0.875 \\
Mean & & & & 0.83 & & & & & 0.96 \\
\hline
\end{tabular}

One natural way to compare two relative rankings is using standard statistical measures; as several authors do in the literature. However, the interpretation should be done quite carefully. A higher average efficiency score in $\mathrm{T} 2$ compared to $\mathrm{T} 1$, does not mean that a specific unit (e.g. C) improved its efficiency seen in an isolated context. In fact, unit $\mathrm{C}$ in $\mathrm{T} 2$ uses a higher amount of inputs to produce the same amount of output. It means is that when $\mathrm{C}$ is compared with the more efficient units $\mathrm{A}$ and $\mathrm{B}$, its efficiency turns out to be closer to them (i.e. become more efficient in the same year). Looking more carefully, we also see that $\mathrm{T} 2$ is a more demanding year for all units, because in general the amount of input needed to maintain the same output has increased.

Therefore, a way to differentiate this to viewpoints is required. To overcome the latest issue, we define an index to measure the effort to obtain input resources in a sequence of years. For that, consider the notation in the previous section. First, we define the Effort Indicator of the set $V$ of units, in the sector $s$ between the period $t_{j-1}$ and the period $t_{j}$, as

$$
E I_{t_{j-1} t_{j}}^{s}(V)=-1+\frac{1}{\bar{I}} \sum_{i \in[\bar{I}]} \frac{\min \left\{x_{i}\left(c, s, t_{j}\right): c \in V\right\}}{\min \left\{x_{i}\left(c, s, t_{j-1}\right): c \in V\right\}}
$$

where $\bar{I}$ is the subset of input indices where both minima are different from zero in the periods $t_{j-1}$ and $t_{j}$. Observe that all inputs are nonnegative and that a zero minimum on the numerator/denominator formally means that such input was introduced/removed in the second period, so it should not be used for the effort comparison. The Accumulated Effort of the set $V$ of units, in the sector s in the time instance $t_{k}$, is defined by

$$
A E I_{t_{k}}^{s}(V)=\sum_{j \in\{1, \ldots, k\}} E I_{t_{j-1} t_{j}}^{s}(V)
$$

For further details, see Section 4 and Figures 3-5.

\section{Hypotheses Analysis and Results}

In this work, the performance of the manufacturing firms according to their sizes is studied. For such, we use the pre-established rules for the size classification, according to the European Commission recommendation (2003/361/EC), firms are classified as micro, small, medium or large using the following criteria. If a firm employs fewer than 250 persons and have either an annual turnover not exceeding 50 million euros or an annual balance sheet total not exceeding 43 million euros, it is included in the group of SMEs (micro, small and medium firms). Within this group, small firms are the ones which employ fewer than 50 persons and whose annual turnover or annual balance sheet total does not exceed 10 million euros. Finally, micro firms are defined as firms which employ fewer than 10 persons and whose annual turnover or annual balance sheet total does not exceed 2 million euros. 
After analysing our dataset, we divided the data in three clusters by the number of employees, using the following criteria:

- Cluster 1 (cl1): firms with number of employees less than 10;

- Cluster 2 (cl2): firms with number of employees bigger or equal than 10 and less than 50 ;

- Cluster 3 (cl3): firms with number of employees greater or equal than 50.

Table 2: The number of firms in each sector by cluster

\begin{tabular}{lrrrrr}
\hline Sector & Cluster 1 & Cluster 2 & Cluster 3 & Total & Eliminated \\
\hline CHEM & 217 & 140 & 46 & 403 & 103 \\
EQUIP & 2,315 & 752 & 197 & 3,264 & 1,056 \\
FOOD & 2,114 & 1,249 & 290 & 3,653 & 1,226 \\
MATER & 1,548 & 804 & 234 & 2,586 & 713 \\
MED & 21 & 18 & 29 & 68 & 17 \\
TEXT & 2,439 & 2,084 & 644 & 5,167 & 1,659 \\
TRANSP & 158 & 102 & 98 & 358 & 104 \\
Total & 8,812 & 5,149 & 1,538 & 15,499 & 4,878 \\
\hline
\end{tabular}

Table 2 relates the cluster groups with the number of firms in each sector, and Figure 1 shows the distribution of the seven manufacturing sectors under study. The last column corresponds to the firms eliminated due to three possible reasons: (i) the obtained dataset does not have or have incorrect values for some variables (i.e. partial information); (ii) the firm does not exist during the entire studied period (i.e. it was established or/and did not survive); or (iii) the database included more firms in the recent years. Unfortunately, we did not find a proper way to distinguish between (ii) and (iii) precisely, nevertheless, the starting point number of firms along years tends to increase, suggesting (iii). Remember that our goal is to analyse the performance of firms, during three years after the intervention of the Troika (2014-2016), for firms which survived the all period.

As we can see in Table 2, more than $50 \%$ of the manufacturing firms in Portugal correspond to firms with a very small number of workers. In fact, the micro firms (cl1) represent $56.8 \%$ of the sample, the share of small firms (cl2) is $33.2 \%$ and the other size firms' (medium or large firms) is $9.9 \%$.

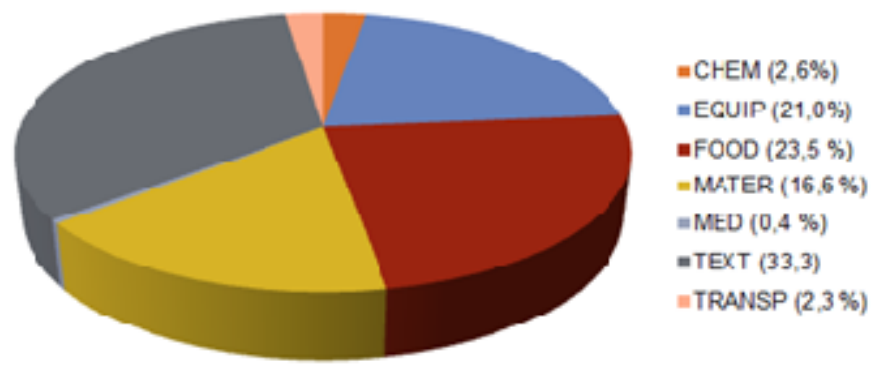

Figure 1: Distribution of the manufacturing firms in 2014-2016 
To continue, we use the standard multivariate technique so-called Principal Component Analysis (PCA), in order to determine the most meaningful input and output variables, by coupling it with a test of dimensionality introduced in Dray (2008). Such approach is used to avoid loss of information (underfitting) or the introduction of random noise (overfitting). The procedure is based on the computation of the RV coefficient, introduced in Robert and Escoufier (1976). From applying PCA and the dimensionality test, we obtain that the "best" variables, in the sense that are more representative/explanatory, that should be considered in the calculation of the efficiency of manufacturing firms. The inputs are: NE, TASSETS, LTDEBT, CLIAB; and the outputs are: PROFITM, LIQR, SOLVR, EBITM, EBITDAM.

\subsection{About the hypothesis (H1): With the intervention of the Troika, the perfor- mance of firms in the manufacturing sector has improved}

For analysing the performance of the firms during the period post-Troika (2014-2016), we examine the different subsectors and try to establish differences in their levels of efficiency. We apply the MEA technique to the nine variables selected in all subsectors. Define EFF as the subset of tuples $n=(c, s, t)$ such that $0.5 \leq M E A Z(n) \leq 1.0$ for a fixed sector $s \in S$, where $M E A Z(n)$ denotes the MEA score (see Equation 1).

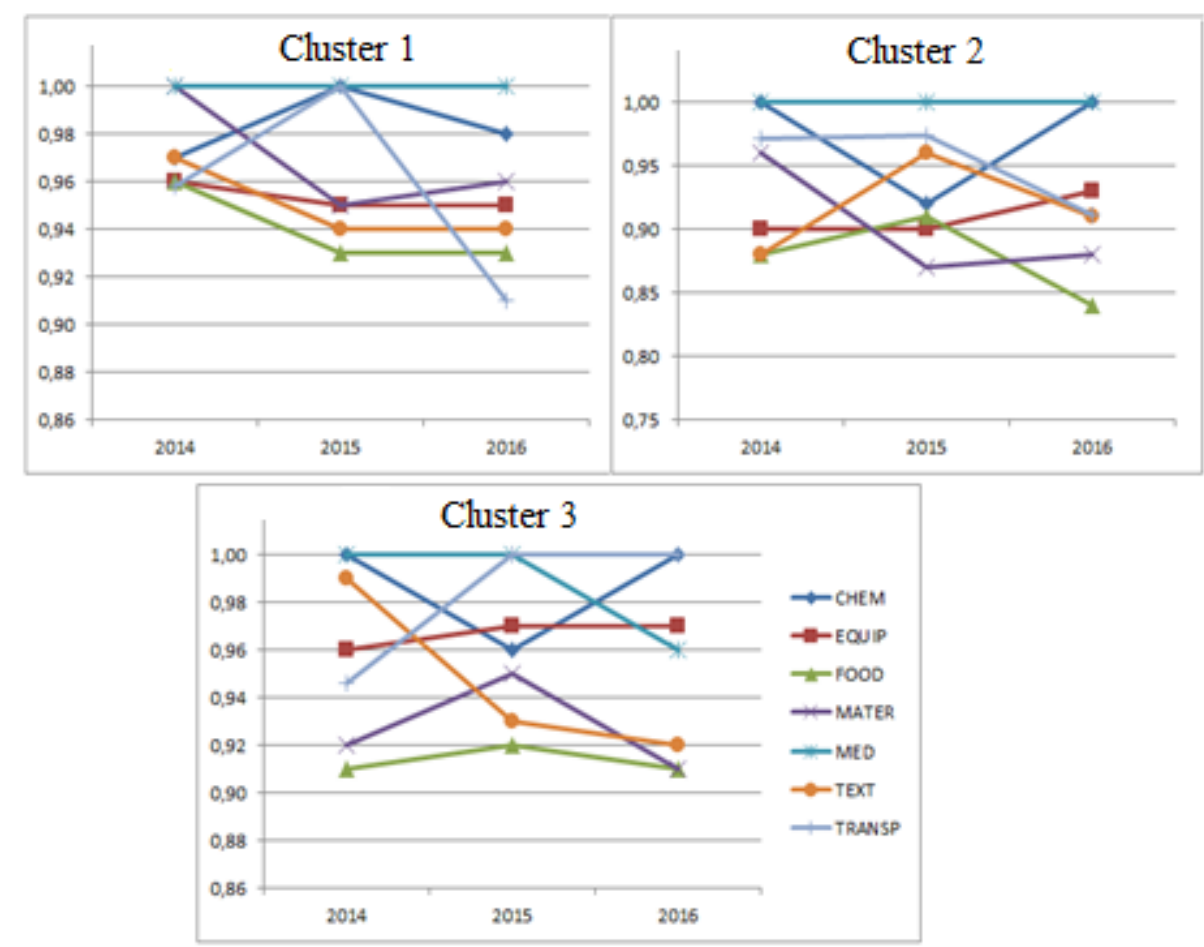

Figure 2: EFF mean values in each cluster

In Figure 2, the EFF mean for each cluster is represented. As we can see, none of the sectors showed a constant improvement, all showed ups and downs. In fact, firms that improved their level of efficiency in 2014, this decreased in the year to follow and vice versa. 
However, the sectors CHEM, MED and TRANSP were consistently more efficient than the other subsectors along the years. Meanwhile, the least efficient subsectors vary depending on the cluster. The subsector MATER remains as one of the most unstable sectors, changing its efficiency level from cluster to cluster. The intention of Figure 2 is showing the distribution of efficiencies above 0.5 by plotting the mean (average) value of EFF, through the years, in order to characterize how the firms of different size were influenced in each sector. Hence, higher values in the plot don't mean that the sector is globally more efficient, since the firms with efficiency below 0.5 are not considered there. It says that the firm's efficiency is more concentrated on the top.

To understand the effort made in each year to acquire inputs, we calculated the accumulated effort indicator AEI (see Equation 6), represented in Figures 3-5. The set of firms used here are the ones with MEA score above 0.75. If the threshold includes the subset of all the more efficient firms, it turns out to be invariant to the choice of value. Since the ideal firm is the one that is able to minimize all the inputs and, at the same time, maximize all the outputs, Figures 3-5 show the effort of each sector to acquire the inputs required to maintain the ideal unit, i.e. theoretically the most efficient firm in the scenario.

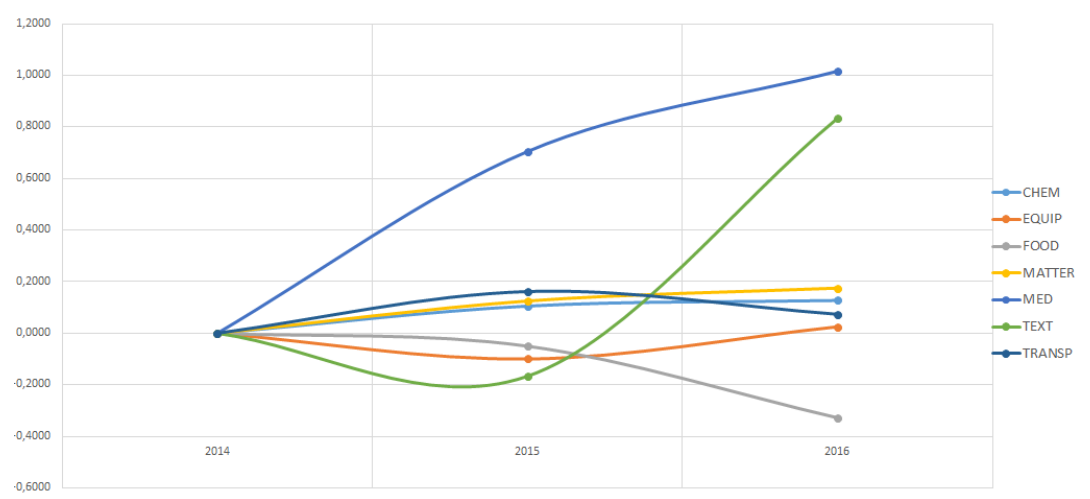

Figure 3: Accumulated effort by sector and cluster 1

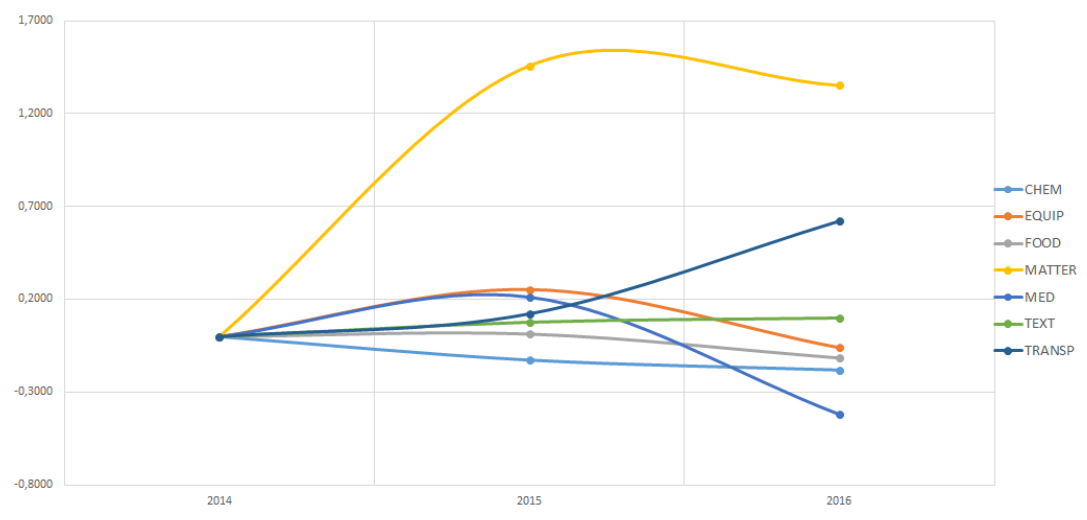

Figure 4: Accumulated effort by sector and cluster 2 


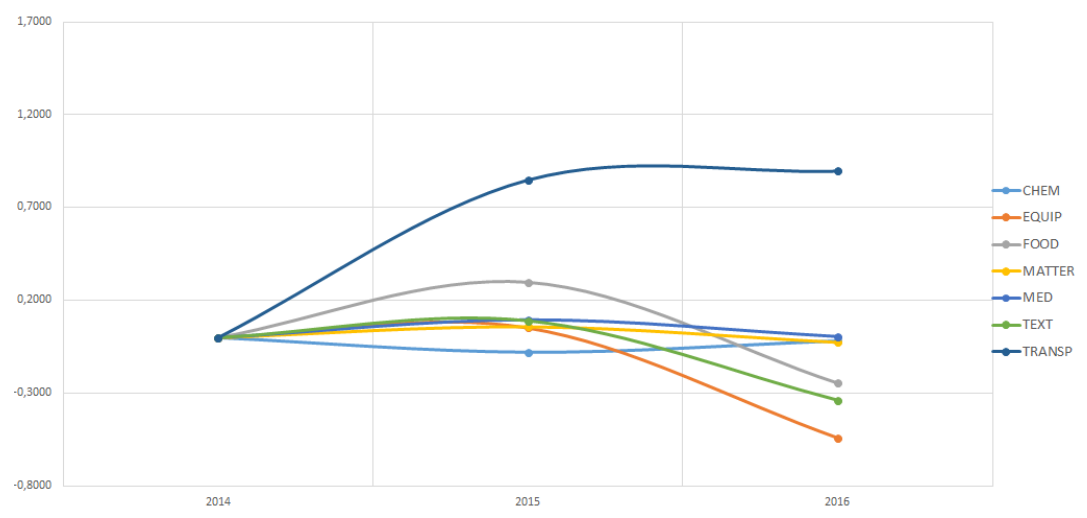

Figure 5: Accumulated effort by sector and cluster 3

As we can see, in general terms, all the companies showed very different behaviours in each sector. The TEXT, TRANSP and MED sectors required more effort than other sectors, to have a good performance in clusters 1, 2 and 3 respectively. In contrast, the MED, TRANSP and MATER sectors showed greater ability to manage their resources in the same clusters.

In Figures 3-5, we can see the importance of the classification of companies. A clear example is the behaviour of the TEXT sector: smaller companies (cluster 1) required a greater effort than other sectors to maintain the ideal unit, see Figure 3. The medium-sized companies (cluster 2) maintained an almost linear behaviour, showing a more balanced control of their resources throughout the study period, see Figure 4. The large companies (cluster 3), reflected great flexibility in the management of their resources, especially in the last year, see Figure 5 .

We may extract three relevant quantities from the efficiency scores. Fixed $s \in[S]$ and $t \in[T]$, we define: efficient firms (EFF) as the percentage of tuples n such $0.5 \leq M E A(n) \leq$ 1.0; fully efficient firms (FullEFF) as the percentage of firms with efficiency equal to 1.0; and null efficient firms (NullEFF) as the percentage of firms with efficiency equal to 0.

Table 3: Efficiency ratios statistics of CHEM and TRANSP subsectors

\begin{tabular}{|c|c|ccc|ccc|}
\hline \multirow{2}{*}{ Cluster } & \multirow{2}{*}{ Year } & \multicolumn{3}{|c|}{ CHEM } & \multicolumn{3}{c|}{ TRANSP } \\
\cline { 3 - 8 } cl1 & EFF & FullEFF & NullEFF & EFF & FullEFF & NullEFF \\
\hline \multirow{3}{*}{ cl2 } & 2014 & 10.1 & 9.7 & 34.1 & 15.2 & 13.9 & 34.2 \\
& 2015 & 7.8 & 7.8 & 26.3 & 7.6 & 7.6 & 30.4 \\
& 2016 & 9.2 & 8.8 & 29.0 & 13.3 & 10.8 & 29.7 \\
\hline \multirow{3}{*}{ cl3 } & 2014 & 7.9 & 7.9 & 25.0 & 15.7 & 14.7 & 18.6 \\
& 2015 & 9.3 & 7.9 & 25.0 & 17.6 & 16.7 & 18.6 \\
& 2016 & 10.0 & 10.0 & 20.7 & 15.7 & 12.7 & 18.6 \\
\hline & 2014 & 28.3 & 28.3 & 17.4 & 18.4 & 16.3 & 14.3 \\
& 2015 & 30.4 & 28.3 & 13.0 & 18.4 & 18.4 & 18.4 \\
& 2016 & 30.4 & 30.4 & 21.7 & 20.4 & 20.4 & 18.4 \\
\hline
\end{tabular}

Analysing the resulting ratios efficiencies, we noticed that it is difficult to establish a behaviour rule. To determine in detail the type of differences that are presented, we compare the MED sector with TRANSP. A summary of the resulting ratios efficiencies of CHEM and 
TRANSP in the three clusters is provided in the Table 3. As we can see, EFF has the highest percentage in cluster 3, in the year 2016 for both sectors. In cluster 1, the two sectors also show a similar behaviour with a better level of efficiency in 2014. For the cluster 2, the ratios are very different from year to year. We point out that the percentage of FullEFF is always lower than NullEFF for cluster 1 (micro firms) and 2 (small firms), but not for cluster 3 (other size firms). Such outcome is a reflection of the fact that, whenever the outputs were negative, the firm received automatically zero efficiency score, which makes sense since the outputs are ratios that whenever have negative values translate into loss or inefficiency in some precise sense. Therefore, the main conclusion is that micro and small firms were badly affected by Troika measures but medium and large firms were able to improve.

\subsection{About the hypothesis (H2)}

The hypothesis (H2) translates to analyse whether during the post-Troika period, LTDEBT was one of the variable more used inefficiently in all subsectors. To this end, we use the inefficiency index (Equation 4).

Detailed results of the inefficiency index for all sectors with respect to the three clusters, are presented in Table 4 . The percentages in the table, represent the number of times each input was used inefficiently (excess inputs).

Table 4: Inefficiency index of the inputs: A (NE), B (TASSETS), C (LTDEBT) and D (CLIAB)

\begin{tabular}{|c|c|c|c|c|c|c|c|c|c|c|c|c|c|}
\hline \multirow[b]{2}{*}{ Year } & \multirow[b]{2}{*}{ Sector } & \multicolumn{4}{|c|}{ Cluster 1} & \multicolumn{4}{|c|}{ Cluster 2} & \multicolumn{4}{|c|}{ Cluster 3} \\
\hline & & A & B & $\mathbf{C}$ & D & A & B & C & D & A & B & $\mathrm{C}$ & D \\
\hline 2014 & & 0.57 & 0.47 & 0.68 & 0.59 & 0.57 & 0.50 & 0.84 & 0.67 & 0.48 & 0.63 & 0.89 & 0.72 \\
\hline 2015 & & 0.65 & 0.63 & 0.88 & 0.78 & 0.56 & 0.54 & 0.81 & 0.64 & 0.39 & 0.14 & 0.88 & 0.21 \\
\hline 2016 & & 0.67 & 0.64 & 0.88 & 0.80 & 0.57 & 0.48 & 0.86 & 0.69 & 0.31 & 0.10 & 0.90 & 0.12 \\
\hline 2014 & & 0.68 & 0.63 & 0.84 & 0.77 & 0.60 & 0.61 & 0.76 & 0.72 & 0.46 & 0.41 & 0.66 & 0.55 \\
\hline 2015 & & 0.68 & 0.53 & 0.71 & 0.62 & 0.63 & 0.62 & 0.75 & 0.73 & 0.55 & 0.57 & 0.79 & 0.69 \\
\hline 2016 & & 0.53 & 0.43 & 0.72 & 0.53 & 0.73 & 0.73 & 0.87 & 0.82 & 0.69 & 0.55 & 0.75 & 0.66 \\
\hline 2014 & & 0.19 & 0.21 & 0.33 & 0.33 & 0.25 & 0.35 & 0.51 & 0.40 & 0.16 & 0.09 & 0.12 & 0.13 \\
\hline 2015 & & 0.21 & 0.07 & 0.42 & 0.04 & 0.31 & 0.53 & 0.81 & 0.56 & 0.19 & 0.18 & 0.43 & 0.22 \\
\hline 2016 & & 0.25 & 0.60 & 0.38 & 0.82 & 0.43 & 0.41 & 0.88 & 0.49 & 0.14 & 0.06 & 0.27 & 0.15 \\
\hline 2014 & & 0.58 & 0.42 & 0.74 & 0.63 & 0.56 & 0.53 & 0.73 & 0.77 & 0.48 & 0.37 & 0.63 & 0.49 \\
\hline 2015 & TRANSP & 0.62 & 0.50 & 0.71 & 0.64 & 0.51 & 0.50 & 0.74 & 0.73 & 0.45 & 0.33 & 0.68 & 0.40 \\
\hline 2016 & & 0.60 & 0.40 & 0.33 & 0.48 & 0.53 & 0.61 & 0.82 & 0.80 & 0.39 & 0.37 & 0.80 & 0.38 \\
\hline 2014 & & 0.69 & 0.73 & 0.90 & 0.81 & 0.60 & 0.56 & 0.55 & 0.66 & 0.50 & 0.54 & 0.85 & 0.62 \\
\hline 2015 & & 0.69 & 0.72 & 0.91 & 0.81 & 0.61 & 0.52 & 0.53 & 0.67 & 0.52 & 0.51 & 0.78 & 0.60 \\
\hline 2016 & & 0.72 & 0.68 & 0.90 & 0.83 & 0.69 & 0.54 & 0.57 & 0.70 & 0.53 & 0.53 & 0.87 & 0.55 \\
\hline 2014 & & 0.75 & 0.65 & 0.94 & 0.68 & 0.73 & 0.53 & 0.88 & 0.56 & 0.45 & 0.46 & 0.56 & 0.45 \\
\hline 2015 & MATER & & 0.47 & 0.81 & 0.5 & 0.67 & 0.52 & 0.91 & 0. & 0.72 & 0.66 & 0.91 & 0.76 \\
\hline 2016 & & 0.66 & 0.47 & 0.81 & 0.57 & 0.71 & 0.63 & 0.86 & 0.65 & 0.49 & 0.46 & 0.53 & 0.49 \\
\hline 2014 & & 0.75 & 0.75 & 0.94 & 0.88 & 0.67 & 0.66 & 0.83 & 0.78 & 0.46 & 0.52 & 0.76 & 0.63 \\
\hline 2015 & TEXT & 0.76 & 0.72 & 0.91 & 0.83 & 0.66 & 0.68 & 0.87 & 0.82 & 0.50 & 0.56 & 0.81 & 0.72 \\
\hline 2016 & & 0.76 & 0.72 & 0.90 & 0.80 & 0.69 & 0.68 & 0.88 & 0.80 & 0.70 & 0.58 & 0.85 & 0.74 \\
\hline
\end{tabular}

As we can see, during the post-Troika period, the three clusters showed again very different behaviours with respect to the variation of the values during years for a fixed subsector. Nevertheless, we may say that, in the three clusters, the variable that is used with less efficiency is LTDEBT, in all years; except for TRANSP in 2016 (cluster 1), for MED in 2014 (cluster 3), and EQUIP (cluster 2), in which the variable that is used with less efficiency is CLIAB in all post-Troika period. This tells us that (H2) cannot be validated 
with respect to the usage of LTDEBT, since the general behaviour is precisely the opposite. Since the other variables do not show a clear trend, it is difficult to establish which variable is the best to use. See, for instance, the inefficiency index for all sectors with respect to cluster 2 in 2014, in Figure 6.

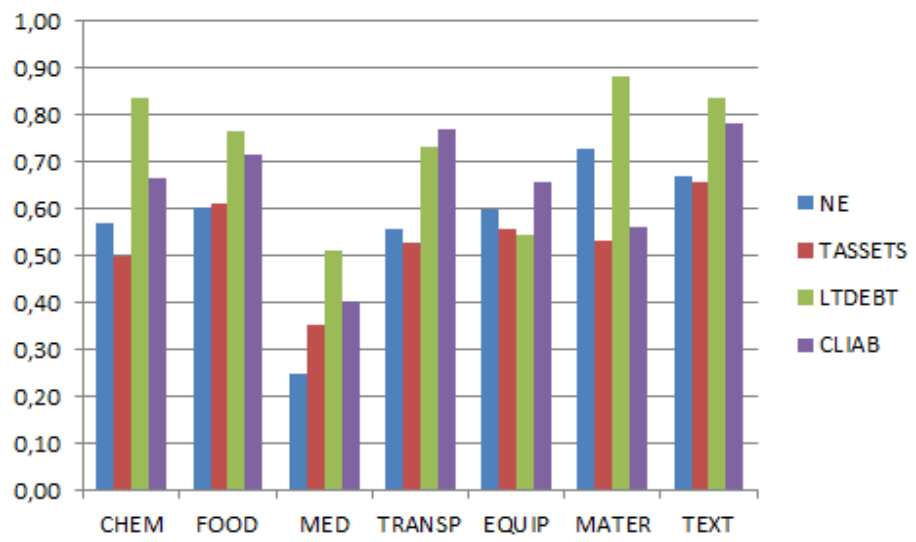

Figure 6: Inefficiency index of the inputs on cluster 2 in 2014

We are interested in analysing the changes after the crises by the subsectors in each cluster to determine if the efficiency patterns present similarities between these subsectors, regarding the acquisition of debt.

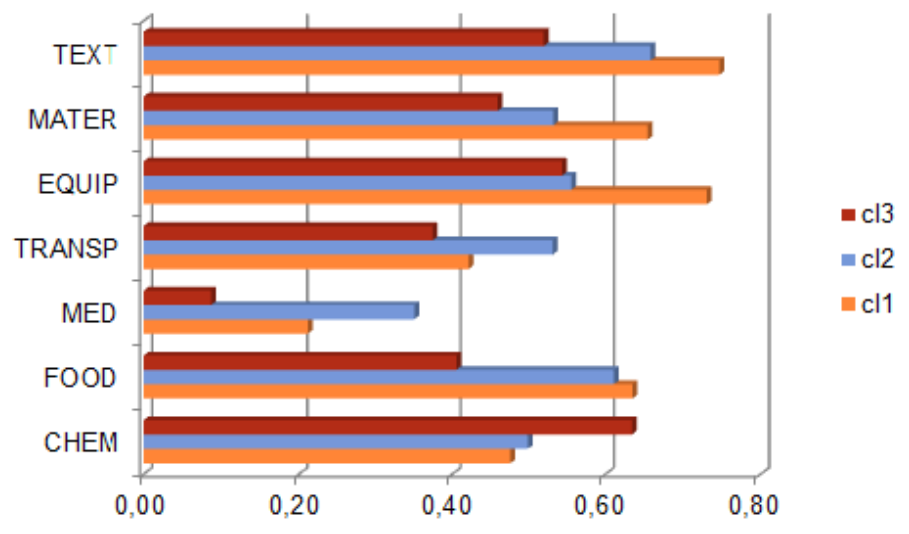

Figure 7: Inefficiency index of LTDEBT in 2014

As mentioned before, LDEBT was the variable most used inefficiently in most subsectors $(77 \%)$, so we may assume that the post-Troika period increased the acquisitions of debt. The proportion of inefficiency of LTDEBT with respect to the clusters for the year 2014, is presented in Figure 7. The idea is comparing the proportion of difference presented in the acquisition of debt in each sector versus the cluster to which they belong. A direct observation is that the micro firms (cl1) borrowed more after the intervention of the Troika, followed by the small firms (cl2). 


\section{Concluding Remarks}

In this work, we examine the effects of the intervention of the Troika on the efficiency scores of $15.5 \mathrm{k}$ firms, obtained from a set of Portuguese manufacturing firms in the postTroika (2014-2016) period. Our study involve all the Portuguese firms of manufacturing industry of Amadeus database, divided in seven sectors.

We adopte the Multidirectional Efficiency Analysis as the main tool to address two empirical hypotheses:

(H1) With the intervention of the Troika, the performance of firms in the manufacturing sector has improved;

(H2) After the financial crisis and corresponding intervention of the Troika, the manufacturing sector significantly acquired long-term debt and used it in an efficient way.

Our results show that the first hypothesis was rejected and the second hypothesis was partially confirmed. As expected, although Portugal has been deeply affected by the global financial crisis, the impact of the recession and subsequent austerity on manufacturing sector has not been uniform. Some sectors have managed to maintain an acceptable level of efficiency, according to the circumstances presented, as long as others have resorted to strategies such as acquisition of a long-term debt, which have offered a momentary solution, although have not produced the best results in their firms in terms of solvency and profit.

On the other hand, in many countries, the crisis and the resulting national austerity measures had a negative impact on labour markets. In general terms, the global financial crisis led to a reduction in the supply of credit, which interferes with firms depending on their size. In fact, small firms differ from large firms in many aspects (taxability, ownership, flexibility, financial market access, etc.) and in this sense it is clear that small firms have a lower financing capacity, which ends up with generating greater impact on them. Our results show that in Portugal, one of the biggest drawbacks is the fact that more than $50 \%$ of the manufacturing firms are micro-firms; but only $9.9 \%$ of them corresponds to the medium and large firms. The bigger firms, reflected some ability to overcome the difficulties presented after the intervention of the Troika, reducing the acquisition of long-term debts but the micro and the small firms had the opposite behaviour. It should be noted that in Portugal, only the manufacture of pharmaceuticals, medicinal chemical and botanical products sector has a greater number of medium and large firms than micro and small firms; but this sector only corresponds to $0.4 \%$ of the manufacturing sectors.

\section{Acknowledgements}

Work partially supported by Portuguese funds through the Centre for Research and Development in Mathematics and Applications (CIDMA) and the Portuguese Foundation for Science and Technology (FCT), within project with reference UID/MAT/04106/2013; funded by Project 3599 - Promover a Produção Científica e Desenvolvimento Tecnológico e a Constituição de Redes Temáticas (3599- PPCDT) and FEDER funds through COMPETE 2020, Programa Operacional Competitividade e Internacionalização (POCI), and by national funds through FCT; Murillo is also supported by the FCT post-doc fellowship with reference SFRH/BPD/97085/2013. 


\section{References}

Abreu, A., Mendes, H., \& Rodrigues, J. (2013). A Crise, a Troika e as Alternativas Urgentes. Tinta-da-China.

Amador, J. (2011). Productivity, Size and Capital Intensity in Selected Portuguese Manufacturing Sectors: A Non-parametric Analysis. Economic Bulletin and Financial Stability Report Articles.

Amador, J., \& Opromolla, L. D. (2009). Textiles and Clothing Exporting Sectors in Portugal - Recent Trends. Banco de Portugal Economic Bulletin Spring, 145-166.

Asmild, M., Baležentis, T., \& Hougaard, J. L. (2016). Multi-directional Program Efficiency: The Case of Lithuanian Family Farms. Journal of Productivity Analysis, 45 (1), 23-33. doi:10.1007/s11123-014-0419-6

Asmild, M., Holvad, T., Hougaard, J. L., \& Kronborg, D. (2009). Railway Reforms: Do They Influence Operating Efficiency? Transportation, 36(5), 617-638. doi:10.1007/s11116009-9216-x

Asmild, M., \& Pastor, J. T. (2010). Slack Free MEA and RDM with Comprehensive Efficiency Measures. Omega, 38(6), 475-483. doi:10.1016/j.omega.2009.12.004

Baležentis, T., \& De Witte, K. (2015). One- and Multi-directional Conditional Efficiency Measurement - Efficiency in Lithuanian family farms. European Journal of Operational Research, 245(2), 612-622. doi:10.1016/j.ejor.2015.01.050

Banker, R. D., Charnes, A., \& Cooper, W. W. (1984). Some Models for Estimating Technical and Scale Inefficiencies in Data Envelopment Analysis. Management science, 30(9), 1078-1092. doi:10.1287/mnsc.30.9.1078

Bi, G., Wang, P., Yang, F., \& Liang, L. (2014). Energy and Environmental Efficiency of China's Transportation Sector: A Multidirectional Analysis Approach. Mathematical Problems in Engineering, 2014. doi:10.1155/2014/539596

Blattner, L., Rebelo, F., \& Farinha, L. (2016). What Holds Investment Back Post-Crisis? Evidence from the 2013 Investment Tax Credit in Portugal [Mimeo].

Bogetoft, P., \& Hougaard, J. L. (1999). Efficiency Evaluations Based on Potential (Non-proportional) Improvements. Journal of Productivity Analysis, 12(3), 233-247. doi:10.1023/A:1007848222681

Bogetoft, P., \& Hougaard, J. L. (2004). Super Efficiency Evaluations Based on Potential Slack. European Journal of Operational Research, 152(1), 14-21. doi:10.1016/S03772217(02)00642-2

Bogetoft, P., \& Otto, L. (2011). Benchmarking with DEA, SFA, and R (Vol. 157). International Series in Operations Research and Management Science, Springer Science \& Business Media.

Broughton, A., \& Welz, C. (2013). Impact of the Crisis on Industrial Relations [European Foundation for the Improvement of Living and Working Conditions (Eurofound), Dublin]. https://www.eurofound.europa.eu/sites/default/files/ef _files/docs/eiro/tn1301019s/tn1301019s.pdf.

Cabral, L. M. (2007). Small Firms in Portugal: A Selective Survey of Stylized Facts, Economic Analysis, and Policy Implications. Portuguese Economic Journal, 6(1), 65-88. doi:10.1007/s10258-007-0018-9 
Charnes, A., Cooper, W. W., \& Rhodes, E. (1978). Measuring the Efficiency of Decision Making Units. European journal of operational research, 2(6), 429-444. doi:10.1016/0377-2217(78)90138-8

Costa, H. A. (2012). From Europe as a Model to Europe as Austerity: The Impact of the Crisis on Portuguese Trade Unions. Transfer: European Review of Labour and Research, 18(4), 397-410. doi:10.1177/1024258912458866

Dray, S. (2008). On the Number of Principal Components: A Test of Dimensionality Based on Measurements of Similarity Between Matrices. Computational Statistics \& Data Analysis, 52(4), 2228-2237. doi:10.1016/j.csda.2007.07.015

Dyson, R. G., Allen, R., Camanho, A. S., Podinovski, V. V., Sarrico, C. S., \& Shale, E. A. (2001). Pitfalls and Protocols in DEA. European Journal of operational research, 132(2), 245-259. doi:10.1016/S0377-2217(00)00149-1

Eichenbaum, M., Rebelo, S., \& de Resende, C. (2016). The Portuguese Crisis and the IMF (Background Paper No. BP/16-02/05). Independent Evaluation Office of the International Monetary Fund.

González, P. (2014). Gender Issues of the Recent Crisis in Portugal. Revue de l'OFCE(2), 241-275. doi:10.3917/reof.133.0241

Gurnani, S. (2016). The Financial Crisis in Portugal: Austerity in Perspective [The Libraries Student Research Prize, Paper 9]. https://preserve.lehigh.edu/library -research-prize/9.

Heyes, J., \& Hastings, T. (2016). Where Now for Flexicurity? Comparing Post-crisis Labour Market Policy Changes in the European Union [Global Political Economy Brief].

Hollingsworth, B., \& Smith, P. (2003). Use of Ratios in Data Envelopment Analysis. Applied Economics Letters, 10(11), 733-735. doi:10.1080/1350485032000133381

Kapelko, M., \& Lansink, A. O. (2017). Dynamic Multi-directional Inefficiency Analysis of European Dairy Manufacturing Firms. European Journal of Operational Research, 257(1), 338-344. doi:10.1016/j.ejor.2016.08.009

Matias, F., Baptista, C., \& Salsa, L. (2015). Capital Structure of Portuguese Manufacturing Industry SMEs: An Analysis with Panel Data. Tourism 83 Management Studies, 11(2), 120-129. doi:10.18089/tms.2015.11215

Matos, F. L. d., Miramontes Carballada, Á., Marques, T. S., \& Ribeiro, D. (2015). Housing in a Time of Crisis: Portugal and Spain an Overview. In Proceeding of the Annual Conference ENHR 2015 Housing and Cities in a Time of Change: Are We Focusing on People? http://hdl.handle.net/10216/80155.

Mohd Suhaimi, N. A. B., de Mey, Y., \& Oude Lansink, A. (2017). Measuring and Explaining Multi-directional Inefficiency in the Malaysian Dairy Industry. British Food Journal, 119(12), 2788-2803. doi:10.1108/BFJ-11-2016-0549

Nogueira, M. C., Afonso, Ó., \& Soukiazis, E. (2017). Skill Premium in Portuguese Manufacturing Industries. Applied Economics Letters, 1-4. doi:10.1080/13504851.2017.1391993

Pearson, K. (1901). LIII. On Lines and Planes of Closest Fit to Systems of Points in Space. The London, Edinburgh, and Dublin Philosophical Magazine and Journal of Science, 2(11), 559-572. doi:10.1080/14786440109462720

Pechankova, K. (2016). Participation of Portuguese Manufacturing Industry in Global Value Chains (Master Thesis). School of Technology and Management of the Polytechnic Institute of Leiria. 
Pedroso, P. (2014). Portugal and the Global Crisis: The Impact of Austerity on the Economy, the Social Model and the Performance of the State (Report of the Project "Social Cohesion and the State in Times of Austerity"). Friedrich-Ebert-Stiftung. http:// library.fes.de/pdf-files/id/10722.pdf.

Petmesidou, M., \& Guillén, A. (2015). Economic Crisis and Austerity in Southern Europe: Threat or Opportunity for a Sustainable Welfare State (European Social Observatory Paper Series No. 18).

Robert, P., \& Escoufier, Y. (1976). A Unifying Tool for Linear Multivariate Statistical Methods: The RV- Coefficient. Journal of the Royal Statistical Society. Series C (Applied Statistics), 25(3), 257-265. doi:10.2307/2347233

Scherr, F. C., \& Hulburt, H. M. (2001). The Debt Maturity Structure of Small Firms. Financial Management, 30(1), 85-111.

Vermoesen, V., Deloof, M., \& Laveren, E. (2013). Long-term Debt Maturity and Financing Constraints of SMEs During the Global Financial Crisis. Small Business Economics, 41(2), 433-448. doi:10.1007/s11187-012-9435-y

Wang, B., Nistor, I., Murty, T., \& Wei, Y.-M. (2014). Efficiency Assessment of Hydroelectric Power Plants in Canada: A Multi Criteria Decision Making Approach. Energy Economics, 46, 112 - 121. doi:10.1016/j.eneco.2014.09.001

Wang, K., Wei, Y.-M., \& Zhang, X. (2013). Energy and Emissions Efficiency Patterns of Chinese Regions: A Multi-directional Efficiency Analysis. Applied Energy, 104, 105 116. doi:10.1016/j.apenergy.2012.11.039

\section{Appendices}

\section{Appendix A.}

The appendix with the description of the Multidirectional Efficiency Analysis model used, is available online on http://sdl-vm2.mathdir.org/docs/_downloads/Appendix.pdf. 